\title{
A change in the timing for starting systemic therapies for hepatocellular carcinoma : the comparison of sorafenib and lenvatinib as the first-line treatment
}

T. Hatanaka', S. Kakizaki², T. Nagashima ${ }^{3}$, T. Ueno ${ }^{4}$, M. Namikawa ${ }^{5}$, H. Tojima ${ }^{2}$, D. Takizawa ${ }^{6}$, A. Naganuma ${ }^{7}$ H. Arai ${ }^{6}$, K. Sato ${ }^{2}$, N. Harimoto ${ }^{8}$, K. Shirabe ${ }^{8}$, T. Uraoka ${ }^{2}$

(1) Department of Gastroenterology, Gunma Saiseikai Maebashi Hospital ; (2) Department of Gastroenterology and Hepatology, Gunma University Graduate School of Medicine ; (3) Department of Gastroenterology, National Hospital Organization Shibukawa Medical Center ; (4) Department of Internal Medicine, Isesaki Municipal Hospital ; (5) Department of Internal Medicine, Kiryu Kosei General Hospital ; (6) Department of Gastroenterology, Maebashi Red Cross Hospital ; (7) Department of Gastroenterology, National Hospital Organization Takasaki General Medical Center ; (8) Department of General Surgical Science, Gunma University Graduate School of Medicine;

\begin{abstract}
Aim : The aim of this retrospective multicenter study was to evaluate the differences in the timing for starting systemic therapies as the first-line treatment for hepatocellular carcinoma (HCC).

Methods: A total of 375 patients with HCC treated with sorafenib from May 2009 to March 2018 and 56 patients treated with lenvatinib from March 2018 to November 2018 at our affiliated hospitals were included in this study.

Results : The median ages of the sorafenib and lenvatinib groups were 71.0 (interquartile range $[I Q R]: 64.0-77.0)$ and 73.5 (IQR : 68.0 -80.0) years old, and $300(80.0 \%)$ and $42(75.0 \%)$ patients were men, respectively. The Barcelona Clinic Liver Cancer stage was early, intermediate and advanced in 39 patients $(10.4 \%), 133$ patients $(35.5 \%)$ and 203 patients $(54.1 \%)$ in the sorafenib group and 1 patient $(1.8 \%), 17$ patients $(30.4 \%)$ and 38 patients $(67.9 \%)$ in the lenvatinib group, respectively. In the analysis of intermediate HCC, patients who satisfied the criteria of TACE failure/ refractoriness $(P=0.017)$, those with ALBI grade $1(P=0.040)$, and those with a serum AFP level $<200 \mathrm{ng} / \mathrm{ml}(\mathrm{P}=\mathbf{0 . 0 2 7})$ were found more frequently in the lenvatinib group than in the sorafenib group, with statistical significance. The objective response rate (ORR) of lenvatinib was $34.8 \%$ in the overall patients and $46.7 \%$ in the intermediate-stage $\mathrm{HCC}$ patients, which was significantly higher than sorafenib $(\mathrm{P}=0.001, \mathrm{P}=0.017)$.

Conclusions: The emergence of lenvatinib has encouraged physicians to start systemic chemotherapy earlier in intermediatestage HCC patients. (Acta gastroenterol. belg., 2021, 84, 65-72).
\end{abstract}

Keywords : Hepatocellular carcinoma, sorafenib, lenvatinib.

\section{Introduction}

Systemic chemotherapy is recommended for treating advanced-stage hepatocellular carcinoma (HCC) patients according to the European Association for the Study of the Liver (EASL) clinical practice guideline (1), whereas transcatheter arterial chemoembolization (TACE) is recommended for intermediate-stage HCC patients. The definition of TACE failure/refractoriness was proposed by the Japan Society of Hepatology (JSH) and the Liver Cancer Study Group of Japan (LCSGJ) (2). Switching from TACE to systemic therapy should be considered in intermediate-stage HCC patients who met the definition of TACE failure/refractoriness (2). Two retrospective cohort studies showed that the overall survival was longer and the liver function better preserved in HCC patients who switched to sorafenib than in those who continued
TACE $(3,4)$. Accordingly, systemic chemotherapy has been recommended for all advanced-stage HCC patients and intermediate-stage $\mathrm{HCC}$ patients who meet the definition of TACE failure/refractoriness in Japan.

Sorafenib (Nexavar ${ }^{\circledR}$, Bayer Pharma AG, Berlin, Germany), an oral multikinase inhibitor, was proven to be associated with a better overall survival in advanced HCC patients $(5,6)$ than the control group and has been widely used as the first-line treatment for advanced HCC patients (1). Recently, an open-label, phase 3, multicenter trial showed that lenvatinib was non-inferior to sorafenib in the overall survival in advanced HCC patients (7). Therefore, lenvatinib is also recommended as a first-line treatment for advanced HCC patients (1).

Lenvatinib (Lenvima ${ }^{\circledR}$; Eisai, Co., Ltd., Tokyo, Japan), which is an oral multikinase inhibitor, inhibits multiple receptor tyrosine kinases, including vascular endothelial growth factor receptors 1-3, fibroblast growth factor receptors 1-4, platelet-derived growth factor receptor $\alpha$, KIT and RET (8-10), and has shown a clinically better objective response than sorafenib (7). However, few studies have investigated the effect of the emergence of lenvatinib on the timing of starting systemic therapy in the clinical setting.

The present study focused on the effects of changing the timing of starting systemic therapy in HCC patients.

\section{Methods}

\section{Inclusion and exclusion criteria}

In this retrospective multicenter study, the inclusion criteria were as follows : (1) HCC diagnosed by typical radiological findings on dynamic enhanced computed tomography $(\mathrm{CT})$ or magnetic resonance imaging (MRI)

\footnotetext{
Correspondence to : Satoru Kakizaki, MD, PhD, Department of Gastroenterology and Hepatology, Gunma University Graduate School of Medicine, 3-39-15 Showa-machi, Maebashi, Gunma 371-8511, Japan.

Tel : +81-27-220-8127. Fax : +81-27-220-8136.

E-mail : kakizaki@gunma-u.ac.jp

Submission date : 07/05/2020

Acceptance date : 22/06/2020
} 


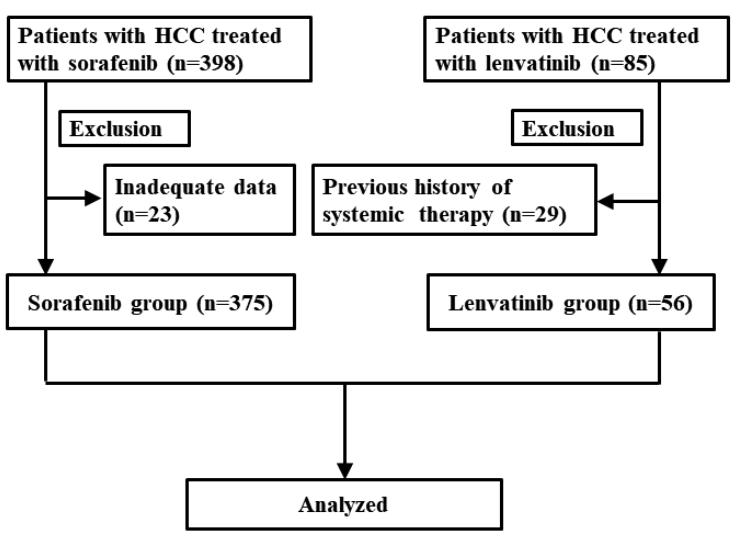

Figure 1. - A flow chart of patients with hepatocellular carcinoma treated with sorafenib or lenvatinib.

(11) or historically proven disease, (2) no indications for surgical resection, liver transplantation or radiofrequency ablation and (3) a liver function of Child-Pugh class A or B. The exclusion criteria were as follows : (1) a history of molecular-targeted therapies and (2) available patient data not adequate.

\section{Patients}

A flow chart of the patient selection process is described in Figure 1. A total of 398 patients with HCC treated with sorafenib from May 2009 to March 2018 and 85 patients treated with lenvatinib from March 2018 to November 2018 treated with lenvatinib (lenvatinib group) were ultimately analyzed.

\section{Treatment}

The decision to treat a patient with sorafenib or lenvatinib was made by the attending physician in daily clinical practice. All treatment decisions, including the timing of treatment, initial dose and duration of therapy, were left to the sole discretion of the attending physician. Sorafenib and lenvatinib were orally administered and continued until the progression of disease or the development of serious adverse events.

\section{Statistical analyses}

Continuous variables were expressed as the median (interquartile range $[\mathrm{IQR}]$ ) and compared using the Mann-Whitney $U$ test. Categorical variables were expressed as the number (percentage) and compared with a chi-squared test or Fisher's exact test if appropriate. The best radiological response was assessed by the modified Response Evaluation Criteria in Solid Tumors (mRECIST). The objective response rate (ORR) was defined as a complete response (CR) or partial response (PR), and the disease control rate (DCR) was defined as a as CR, PR or stable disease (SD). The criteria of TACE failure/refractoriness were based on the proposal of the JSH-LCSGJ (2). The up-to-seven criteria were defined as the sum of the maximum tumor diameter in liver (in $\mathrm{cm}$ ) and the number of tumors $\leq 7$ in patients with intermediate-stage HCC (12). We calculated the albumin-bilirubin (ALBI) score using the following formula : ALBI score : $(\log 10$ bilirubin $[\mu \mathrm{mol} / \mathrm{L}] \times 0.66)$ $+($ albumin $[\mathrm{g} / \mathrm{L}] \times-0.085)$, and the ALBI grade was defined by the resulting score $(\leq-2.60=$ grade $1,>-2.60$ to $-1.39=$ grade $2,>-1.39=$ grade 3$)$ (13). The overall survival was estimated by the Kaplan-Meier method, and the log-rank test was used to compare the two groups.

Five patients in the sorafenib group had missing data for the AFP analysis, and 9 patients in the sorafenib group and 1 patient in the lenvatinib group had missing data for the DCP analysis. We excluded 70 patients in the sorafenib group and 10 patients in the lenvatinib group from the analysis of the best radiological response due to a lack of evaluable radiological imaging.

This study was conducted according to the ethical guidelines of the Declaration of Helsinki and approved by the Ethics Review Committee of each institution. The need for written informed consent was waived because of the retrospective nature of the study. All statistical analyses were done by the IBM Statistical Package for the Social Sciences software version 24 (IBM SPSS 24, IBM, Armonk, USA).

\section{Results}

The median ages of the sorafenib and lenvatinib groups were 71.0 (IQR : 64.0-77.0) and 73.5 (IQR : 68.0$80.0)$ years old, and $300(80.0 \%)$ and $42(75.0 \%)$ patients were men, respectively. The most frequent etiology of underlying liver diseases was hepatitis $\mathrm{C}$ virus (HCV) in both groups. Child-Pugh class was A and B in 304 $(81.1 \%)$ and $71(18.9 \%)$ patients in the sorafenib group and in $50(89.3 \%)$ and $6(10.7 \%)$ patients in the lenvatinib group, respectively. The ALBI grade was 1, 2 and 3 in $90(24.0 \%), 265(70.7 \%)$ and $20(5.3 \%)$ patients in the sorafenib group and in $19(33.9 \%), 37$ (66.1\%) and $0(0.0 \%)$ patient in the lenvatinib group, respectively. With respect to the clinical stage, the Barcelona Clinic Liver Cancer stage was early, intermediate and advanced in 39 patients $(10.4 \%), 133$ patients $(35.5 \%)$ and 203 patients $(54.1 \%)$ in the sorafenib group and 1 patient (1.8\%), 17 patients $(30.4 \%)$ and 38 patients $(67.9 \%)$ in the lenvatinib group, respectively. The age of lenvatinib group was significantly older than that in sorafenib group $(\mathrm{P}=0.038)$. There were no significant differences in other factors between the two groups. This information is shown in Table 1.

Concerning the best radiological response in all patients, CR, PR, SD and progression disease (PD) were noted in 5 patients $(1.6 \%), 36$ patients $(11.8 \%)$, 131 patients $(43.1 \%)$ and 133 patients $(43.8 \%)$ in the sorafenib group and 0 patient $(0.0 \%), 16$ patients (34.8\%), 20 patients $(43.5 \%)$ and 10 patients $(21.7 \%)$ in the lenvatinib group, respectively. Accordingly, the ORR and DCR in the lenvatinib group were $34.8 \%$ and $78.3 \%$, 
Table 1. - Patient characteristics of the sorafenib group and lenvatinib group

\begin{tabular}{|c|c|c|c|}
\hline & Sorafenib group $(n=375)$ & Lenvatinib group $(\mathrm{n}=56)$ & P-value \\
\hline Age (years) & $71.0(64.0-77.0)$ & $73.5(68.0-80.0)$ & 0.038 \\
\hline Males, $\mathrm{n}(\%)$ & $300(80.0)$ & $42(75.0)$ & 0.38 \\
\hline Underlying liver disease, $\mathrm{n}(\%)$ & & & 0.31 \\
\hline HBV & $40(10.7)^{*}$ & $3(5.4)$ & \\
\hline $\mathrm{HCV}$ & $224(59.7)^{*}$ & $32(57.1)$ & \\
\hline Alcohol & $40(10.7)$ & $10(17.9)$ & \\
\hline Others & $72(19.2)$ & $11(19.6)$ & \\
\hline Child-Pugh, n (\%) & & & 0.13 \\
\hline A & $304(81.1)$ & $50(89.3)$ & \\
\hline B & $71(18.9)$ & $6(10.7)$ & \\
\hline ALBI grade & & & 0.081 \\
\hline Grade 1 & $90(24.0)$ & $19(33.9)$ & \\
\hline Grade 2 & $265(70.7)$ & $37(66.1)$ & \\
\hline Grade 3 & $20(5.3)$ & $0(0.0)$ & \\
\hline Naïve, n (\%) & $95(25.3)$ & $12(21.4)$ & 0.53 \\
\hline BCLC, n (\%) & & & 0.052 \\
\hline Early & $39(10.4)$ & $1(1.8)$ & \\
\hline Intermediate & $133(35.5)$ & $17(30.4)$ & \\
\hline Advanced & $203(54.1)$ & $38(67.9)$ & \\
\hline $\operatorname{AFP}(\mathrm{ng} / \mathrm{ml}), \mathrm{n}(\%) * *$ & & & 0.18 \\
\hline$<200$ & $214(57.1)$ & $37(67.3)$ & \\
\hline$\geq 200$ & $156(41.6)$ & $18(32.7)$ & \\
\hline $\mathrm{DCP}(\mathrm{mAU} / \mathrm{ml}), \mathrm{n}(\%) * * *$ & & & 0.21 \\
\hline$<200$ & $180(49.2)$ & $32(58.2)$ & \\
\hline$\geq 200$ & $186(50.8)$ & $23(41.8)$ & \\
\hline
\end{tabular}

Continuous variables were expressed as the median (interquartile range). Categorical variables were expressed as the number (percentage). *One patient was positive in both HBs-Ag and HCV-ab. ${ }^{* *}$ There were missing data in 5 patients in the sorafenib group. ${ }^{* * *}$ There were missing data in 9 patients in the sorafenib group and 1 patient in the lenvatinib group.

Table 2. - Best radiological response

\begin{tabular}{|c|c|c|c|}
\hline & Sorafenib group $(\mathrm{n}=305)$ & Lenvatinib group $(\mathrm{n}=46)$ & P-value \\
\hline Best radiological response, $\mathrm{n}(\%)$ & & & $<0.001$ \\
\hline CR & $5(1.6)$ & $0(0.0)$ & \\
\hline PR & $36(11.8)$ & $16(34.8)$ & \\
\hline SD & $131(43.0)$ & $20(43.5)$ & \\
\hline PD & $133(43.8)$ & $10(21.7)$ & 0.001 \\
\hline Response rate (\%) & 13.4 & 34.8 & 0.005 \\
\hline Disease control rate (\%) & 56.4 & 78.3 & \\
\hline
\end{tabular}

Data for both groups were presented as the number (percentage). CR : complete response, PD : progressive disease, PR : partial response, $\mathrm{SD}$ : stable disease.

respectively, which was significantly better than those in the sorafenib group $(\mathrm{P}=0.001, \mathrm{P}=0.005$ ) (Table 2).

At the time of the analysis, 274 patients in the sorafenib group and 19 in the lenvatinib group had died. The median survival time in all patients was estimated to be 14.0 months (95\% confidence interval [CI] 12.3-15.6 months), and the 1-, 2-, 3- and 5-year survival rates were calculated to be $55.0 \%$ (95\% CI, $49.9 \%-60.1 \%), 28.3 \%$ (95\% CI, 23.0\%-33.6\%), 15.8\% (95\% CI, 11.3\%-20.2\%) and $6.8 \%(95 \% \mathrm{CI}, 2.9 \%-10.7 \%)$, respectively (Figure 2a). The median follow-up period was 10 months (IQR : 4.7-19.2 months) in the sorafenib group and 9.8 months (IQR : 4.4-13.3 months) in the lenvatinib group. The median survival time (MST) in the sorafenib group was estimated to be 13.4 months ( $95 \%$ CI, 11.7-15.2 months) whereas the MST in the lenvatinib group was not yet reached. There were no significant differences between the sorafenib group and lenvatinib group $(\mathrm{P}=0.21)$ (Figure 2b).

In the analysis of intermediate-stage HCC patients, the median age was 73.0 (IQR : 64.5-78.0) years old in the sorafenib group and 74.0 (IQR : 67.5-81.0) years old in the lenvatinib group. About $75 \%$ of patients were male in both groups. The ALBI grade in the lenvatinib group was significantly better than that in the sorafenib group $(\mathrm{P}=0.040)$. The number of tumors was $\geq 4$ in 118 patients $(88.7 \%)$ in the sorafenib group and 13 patients $(76.5 \%)$ in the lenvatinib group, without statistical significance $(\mathrm{P}=0.24)$. The median maximum tumor diameter in the liver was $3.1(2.0-5.5) \mathrm{cm}$ in the sorafenib group and 


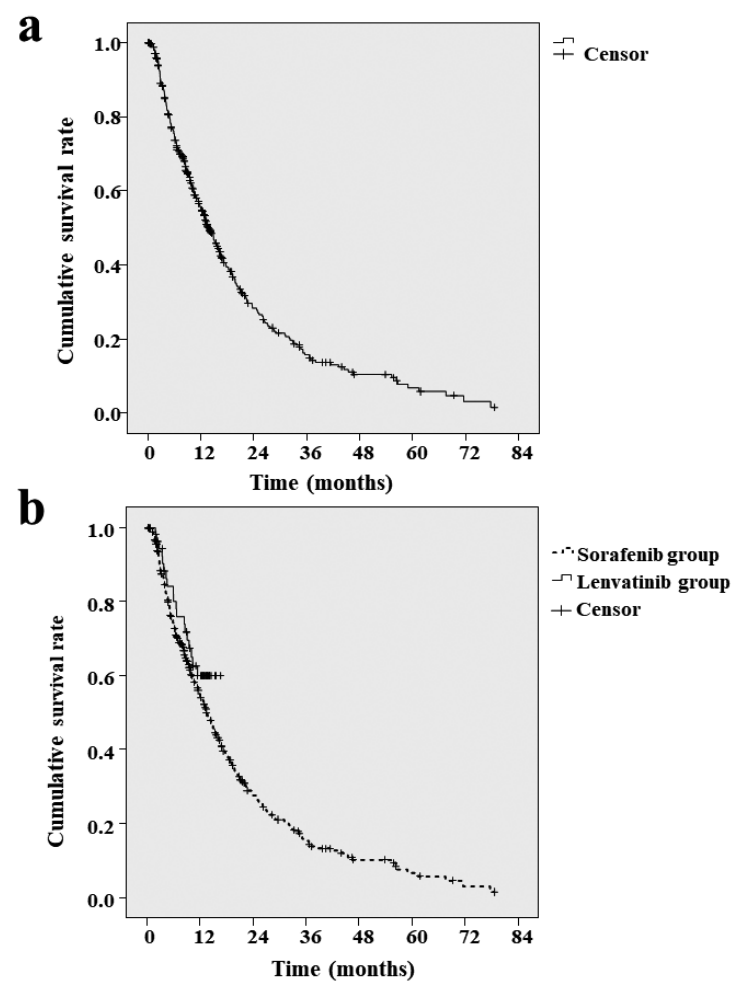

Figure 2. - A Kaplan-Meier curve showed that the median survival time in all patients was estimated to be 14.0 months (95\% confidence interval [CI] 12.3-15.6 months), and the 1-, 2-, 3- and 5-year survival rates were 5.0\% (95\% CI, 49.9\%$60.1 \%$ ), $28.3 \%$ (95\% CI, $23.0 \%-33.6 \%$ ), $15.8 \%$ (95\% CI, $11.3 \%-20.2 \%$ ) and $6.8 \%(95 \%$ CI, $2.9 \%-10.7 \%)$, respectively (a). The median follow-up period was 10 months $(95 \% \mathrm{CI}, 4.7-$ 19.2 months) in the sorafenib group and 9.8 months $(95 \% \mathrm{CI}$, 4.4-13.3 months) in the lenvatinib group. The median survival time (MST) in the sorafenib group was estimated to be 13.4 months (95\% CI, 11.7-15.1 months) whereas the MST in the lenvatinib group was not yet reached. There was not significant difference between the sorafenib group and lenvatinib group $(\mathrm{P}=0.21)(\mathrm{b})$.

$2.0(1.1-6.2) \mathrm{cm}$ in the lenvatinib group $(\mathrm{P}=0.21)$. The patients who met the up-to-seven criteria were 26 patients $(19.5 \%)$ in the sorafenib group and 7 patients (41.2\%) in the lenvatinib group, without statistical significance $(\mathrm{P}=0.06)$. While there was no significant difference in the number of previous TACE treatments between the both groups ( $\mathrm{P}=0.91)$, the percentage of patients who met the criteria of TACE failure/refractoriness in the lenvatinib group was greater than in the sorafenib group $(\mathrm{P}=0.017)$. Patients with a serum AFP level $<200 \mathrm{ng} / \mathrm{ml}$ were found more frequently in the lenvatinib group than in the sorafenib group $(\mathrm{P}=0.027)$ (Table 3 ).

Regarding the timing of starting systemic therapy in intermediate-stage HCC patients, the lenvatinib was started earlier than sorafenib. Because treatment strategies for HCC change with the times, we divided the sorafenib group to early period and late period. Because the concept of TACE failure/refractoriness was first reported in 2014 (2), early sorafenib group and late sorafenib group were defined the term from 2008 to

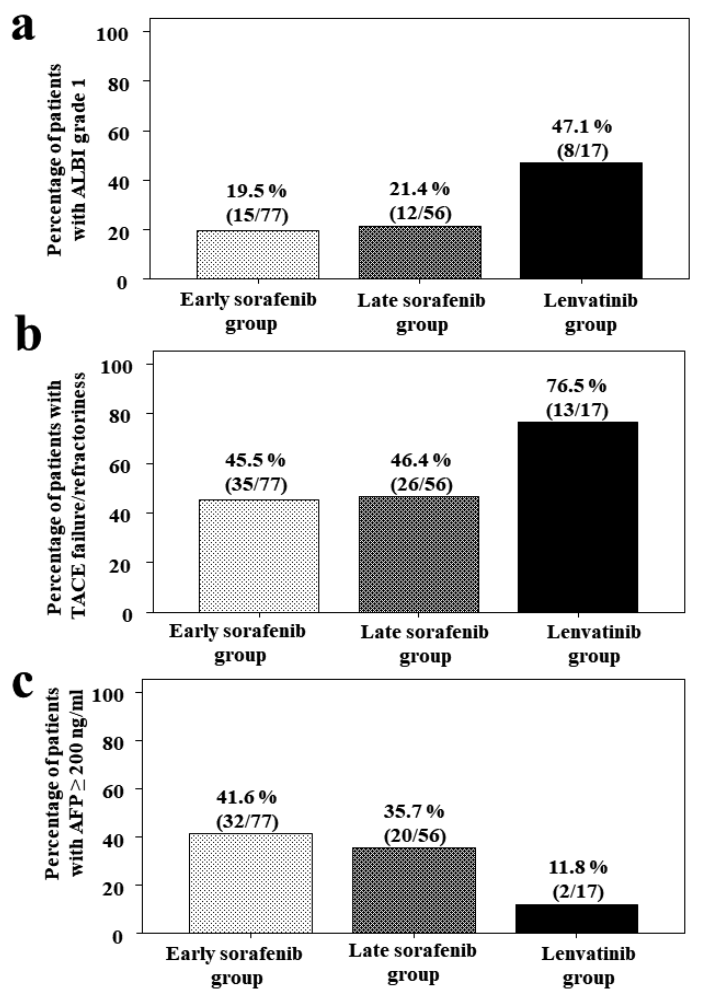

Figure 3. - The percentage of patients with intermediate-stage HCC in early sorafenib, late sorafenib and lenvatinib group. $\mathrm{a}:$ the percentage of patients with the ALBI grade $1, b:$ the percentage of patients who met the criteria of TACE failure/ refractoriness, $\mathrm{c}$ : the percentage of patients with a serum AFP level $\geq 200 \mathrm{ng} / \mathrm{ml}$.

2014 and the term from 2015 to 2018, respectively. Early sorafenib group included 77 patients and late sorafenib group included 56 patients, respectively (Table 4 ). The ALBI grade in the lenvatinib group was significantly better than that in the late sorafenib group $(\mathrm{P}=0.038)$. The percentage of patients who met the criteria of TACE failure/refractoriness in the lenvatinib group was greater than that in the late sorafenib group $(\mathrm{P}=0.030)$. As a result, lenvatinib was started earlier in the patients with TACE failure/refractoriness in comparison with sorafenib. Figure 3 showed the percentage of patients with intermediate-stage $\mathrm{HCC}$ in each group. The percentages of patients with the ALBI grade 1, and those who met the criteria of TACE failure/refractoriness were significantly increased in lenvatinib group. The percentage of patients with a serum AFP level $\geq 200 \mathrm{ng} /$ $\mathrm{ml}$ decreased in lenvatinib group.

The best radiological response in intermediate $\mathrm{HCC}$ patients was $\mathrm{CR}, \mathrm{PR}, \mathrm{SD}$ and $\mathrm{PD}$ in 0 patients $(0.0 \%)$, 7 patients $(46.7 \%), 7$ patients $(46.7 \%)$ and 1 patients $(6.7 \%)$ in the lenvatinib group and 2 patients (1.8\%), 18 patients $(15.9 \%), 36$ patients $(31.9 \%)$ and 57 patients $(50.4 \%)$ in the sorafenib group. Thus, the ORR and DCR in the lenvatinib group were $46.7 \%$ and $93.3 \%$, respectively, which was significantly better than those in the sorafenib group $(\mathrm{P}=0.017, \mathrm{P}=0.001)$ (Table 5). Table 6 showed the best radiological response in patients with 
Table 3. - Characterstics of patients with intermediate-stage HCC

\begin{tabular}{|c|c|c|c|}
\hline & Sorafenib group $(\mathrm{n}=133)$ & Lenvatinib group $(\mathrm{n}=17)$ & P-value \\
\hline Age (years) & $73.0(64.5-78.0)$ & $74.0(67.5-81.0)$ & 0.29 \\
\hline Males, n (\%) & $100(75.2)$ & $13(76.5)$ & 1.00 \\
\hline Underlying liver disease, $\mathrm{n}(\%)$ & & & 0.99 \\
\hline HBV & $15(11.3)$ & $2(11.8)$ & \\
\hline $\mathrm{HCV}$ & $83(62.4)$ & $10(58.8)$ & \\
\hline Alcohol & $14(10.5)$ & $2(11.8)$ & \\
\hline Others & $21(15.8)$ & $3(17.6)$ & \\
\hline Child-Pugh, n (\%) & & & 0.31 \\
\hline A & $110(82.7)$ & $16(94.1)$ & \\
\hline B & $23(17.3)$ & $1(5.9)$ & \\
\hline ALBI grade, $\mathrm{n}(\%)$ & & & 0.040 \\
\hline Grade 1 & $27(20.3)$ & $8(47.1)$ & \\
\hline Grade 2 & $100(75.2)$ & $9(52.9)$ & \\
\hline Grade 3 & $6(4.5)$ & $0(0.0)$ & \\
\hline Naïve, n (\%) & $18(13.5)$ & $3(17.6)$ & 0.71 \\
\hline Number of tumors, n (\%) & & & 0.24 \\
\hline 2,3 & $15(11.3)$ & $4(23.5)$ & \\
\hline$\geq 4$ & $118(88.7)$ & $13(76.5)$ & \\
\hline Maximum tumor diameter in the liver & $3.1(2.0-5.5)$ & $2.0(1.1-6.2)$ & 0.21 \\
\hline Up-to-seven criteria, n (\%) & $26(19.5)$ & $7(41.2)$ & 0.06 \\
\hline Number of previous TACE treatment & $3(1-5)$ & $2(1-8)$ & 0.91 \\
\hline $0-2, \mathrm{n}(\%)$ & $58(43.6)$ & $10(58.8)$ & 0.24 \\
\hline$\geq 3, \mathrm{n}(\%)$ & $75(56.4)$ & $7(41.2)$ & \\
\hline Criteria of TACE failure/refractoriness satisfied, n (\%) & $61(45.9)$ & $13(76.5)$ & 0.017 \\
\hline AFP (ng/ml), n (\%) & & & 0.027 \\
\hline$<200$ & $81(60.9)$ & $15(88.2)$ & \\
\hline$\geq 200$ & $52(39.1)$ & $2(11.8)$ & \\
\hline $\mathrm{DCP}(\mathrm{mAU} / \mathrm{ml}), \mathrm{n}(\%) *$ & & & 0.16 \\
\hline$<200$ & $69(52.7)$ & $12(70.6)$ & \\
\hline$\geq 200$ & $62(47.3)$ & $5(29.4)$ & \\
\hline
\end{tabular}

Continuous variables expressed by median (interquartile range [IQR]). Categorical variables expressed by count and percentage. *There were missing data in 2 patient in sorafenib group. AFP $: \alpha$-fetoprotein, ALBI grade : albumin-bilirubin grade, DCP $:$ des- $\gamma$-carboxy prothrombin, HBV : hepatitis B virus, $\mathrm{HCC}$ : hepatocellular carcinoma, $\mathrm{HCV}$ : hepatitis $\mathrm{C}$ virus, TACE : transcatheter arterial chemoemobolization.

intermediate-stage HCC in early sorafenib, late sorafenib and lenvatinib group.

\section{Discussion}

Our study showed that patients who satisfied the criteria of TACE failure/refractoriness and those with a serum AFP level $<200 \mathrm{ng} / \mathrm{ml}$ were found more frequently in the lenvatinib group than in the sorafenib group in our analysis of intermediate HCC patients, whereas there was not significant differences between the two groups in the analysis of all patients.

The current standard treatment for intermediate HCC is TACE (1). The ORR of TACE was $28 \%-52 \%$ for intermediate $\mathrm{HCC}$ patients according to recent randomized control trials $(14,15)$. In contrast, only $13.9 \%$ subsequently responded to TACE among HCC patients who met the definition of TACE failure/refractoriness (3). Accordingly, switching treatment from TACE to systemic chemotherapy was recommended for intermediate HCC patients with TACE failure/refractoriness (2).

Yasui et al. reported that the up-to-seven criteria was a favorable factor associated with downstaging to within the Milan criteria and preserving the liver function (16). The Japanese HCC expert meeting revealed that patients who would benefit from TACE included those with 4-7 nodules of 3-4 cm in size (2). Accordingly, a better response could be more easily obtained in patients with fewer and smaller tumors than in those with more and larger lesions. In the present study, the lenvatinib group was less advanced than the sorafenib group in the analysis of intermediate-stage HCC patients. Regarding tumor factors, TACE was more favorably indicated for the tumor factors in the lenvatinib group than for those in the sorafenib group. However, repeated TACE worsens the liver function (17), and TACE benefit consisted of the tumor response and preserving the liver function (2). Therefore, lenvatinib treatment was introduced earlier before the liver function had been worsened by repeated TACE, especially in patients who met the criteria of TACE failure/refractoriness.

Sorafenib is a tyrosine kinase inhibitor that was the only available first-line treatment in Japan from May 2009 to March 2018. Previous studies (7,18-21) have reported that the ORR of sorafenib treatment was $8.4 \%$ $22.8 \%$, as evaluated by mRECIST, which was consistent 
Table 4. - Characterstics of patients with intermediate-stage HCC in early sorafenib, late sorafenib and lenvatinib group

\begin{tabular}{|c|c|c|c|c|}
\hline & $\begin{array}{l}\text { Early sorafenib group } \\
(\mathrm{n}=77)\end{array}$ & $\begin{array}{l}\text { Late sorafenib group } \\
\qquad(\mathrm{n}=56)\end{array}$ & $\begin{array}{l}\text { Lenvatinib group } \\
\quad(\mathrm{n}=17)\end{array}$ & P-value \\
\hline Age (years) & $72(63.5-78.0)$ & $74(67.5-78.5)$ & $74(67.5-81.0)$ & 0.59 \\
\hline Males, n (\%) & $59(76.6)$ & $41(73.2)$ & $13(76.5)$ & 1.00 \\
\hline Underlying liver disease, n (\%) & & & & 0.90 \\
\hline HBV & $7(9.1)$ & $8(14.3)$ & $2(11.8)$ & \\
\hline $\mathrm{HCV}$ & $54(70.1)$ & $29(51.8)$ & $10(58.8)$ & \\
\hline Alcohol & $9(11.7)$ & $5(8.9)$ & $2(11.8)$ & \\
\hline Others & $7(9.1)$ & $14(25.0)$ & $3(17.6)$ & \\
\hline Child-Pugh, n (\%) & & & & 0.67 \\
\hline A & $61(79.2)$ & $49(87.5)$ & $16(94.1)$ & \\
\hline $\mathrm{B}$ & $16(20.8)$ & $7(12.5)$ & $1(5.9)$ & \\
\hline ALBI grade, $\mathrm{n}(\%)$ & & & & 0.038 \\
\hline Grade 1 & $15(19.5)$ & $12(21.4)$ & $8(47.1)$ & \\
\hline Grade 2 & $56(72.7)$ & $44(78.6)$ & $9(52.9)$ & \\
\hline Grade 3 & $6(7.8)$ & $0(0.0)$ & $0(0.0)$ & \\
\hline Naïve, n (\%) & $8(10.4)$ & $10(17.9)$ & $3(17.6)$ & 1.00 \\
\hline Number of tumors, $\mathrm{n}(\%)$ & & & & 0.23 \\
\hline 2,3 & $9(11.7)$ & $6(10.7)$ & $4(23.5)$ & \\
\hline$\geq 4$ & $68(88.3)$ & $50(89.3)$ & $13(76.5)$ & \\
\hline Maximum tumor diameter in the liver & $2.9(2.0-5.2)$ & $3.5(2.0-5.7)$ & $2.0(1.1-6.2)$ & 0.29 \\
\hline Up-to-seven criteria, $\mathrm{n}(\%)$ & $15(19.5)$ & $11(19.6)$ & $7(41.2)$ & 0.11 \\
\hline Number of previous TACE treatment & $3(1-5)$ & $3(1-5)$ & $2(1-8)$ & 0.87 \\
\hline $0-2, \mathrm{n}(\%)$ & $31(40.3)$ & $27(48.2)$ & $10(58.8)$ & 0.44 \\
\hline$\geq 3, \mathrm{n}(\%)$ & $46(59.7)$ & $29(51.8)$ & $7(41.2)$ & \\
\hline $\begin{array}{l}\text { Criteria of TACE } \\
\text { failure/refractoriness satisfied, n (\%) }\end{array}$ & $35(45.5)$ & $26(46.4)$ & $13(76.5)$ & 0.030 \\
\hline $\operatorname{AFP}(\mathrm{ng} / \mathrm{ml}), \mathrm{n}(\%)$ & & & & 0.059 \\
\hline$<200$ & $45(58.4)$ & $36(64.3)$ & $15(88.2)$ & \\
\hline$\geq 200$ & $32(41.6)$ & $20(35.7)$ & $2(11.8)$ & \\
\hline $\mathrm{DCP}(\mathrm{mAU} / \mathrm{ml}), \mathrm{n}(\%) * *$ & & & & 0.11 \\
\hline$<200$ & $42(56.0)$ & $27(48.2)$ & $12(70.6)$ & \\
\hline$\geq 200$ & $33(44.0)$ & $29(51.8)$ & $5(29.4)$ & \\
\hline
\end{tabular}

Continuous variables expressed by median (interquartile range [IQR]). Categorical variables expressed by count and percentage. *Statistical analysis was carried out between late sorafenib group and lenvatinib group. **There were missing data in 2 patient in late sorafenib group. AFP : $\alpha$-fetoprotein, ALBI grade : albumin-bilirubin grade, DCP : des- $\gamma$-carboxy prothrombin, HBV : hepatitis B virus, HCC : hepatocellular carcinoma, HCV : hepatitis C virus, TACE : transcatheter arterial chemoemobolization.

Table 5. - Best radiological response in patients with intermediate-stage HCC

\begin{tabular}{|c|c|c|c|}
\hline & Sorafenib group $(\mathrm{n}=113)$ & Lenvatinib group $(\mathrm{n}=15)$ & P-value \\
\hline Best radiological response, $\mathrm{n}(\%)$ & & & 0.004 \\
\hline CR & $2(1.8)$ & $0(0.0)$ & \\
\hline PR & $18(15.9)$ & $7(46.7)$ & \\
\hline SD & $36(31.9)$ & $7(46.7)$ & \\
\hline PD & $57(50.4)$ & $1(6.7)$ & \\
\hline Response rate (\%) & 17.7 & 46.7 & 0.017 \\
\hline Disease control rate (\%) & 49.6 & 93.3 & 0.001 \\
\hline
\end{tabular}

Data for both groups were presented as the number (percentage). CR : complete response, HCC : hepatocellular carcinoma, PD : progressive disease, PR : partial response, SD : stable disease.

with the results of this study. During the sorafenib era, about $46 \%$ of patients with intermediate HCC met the definition of TACE failure/refractoriness based on the results of this study. This relative low percentage may be due in part to various new TACE techniques, such as balloon-occluded TACE $(22,23)$ and drug-eluting beads TACE $(24,25)$, being developed during this period. These new techniques might have encouraged physicians to conduct repeated TACE procedures. Lenvatinib, which was a higher ORR than sorafenib (7), has been allowed to be used as the first-line treatment in Japan since March 2018. Our analysis of intermediate-stage HCC patients in the present study suggested that the emergence of lenvatinib encouraged physicians to convert from TACE to systemic chemotherapy earlier. 
Table 6. - Best radiological response in patients with intermediate-stage HCC in early sorafenib, late sorafenib and lenvatinib group

\begin{tabular}{|l|c|c|c|c|}
\hline & Early sorafenib & Late sorafenib & Lenvatinib group & \multirow{2}{*}{ P-value } \\
\hline & group $(\mathrm{n}=77)$ & group $(\mathrm{n}=56)$ & $(\mathrm{n}=17)$ & 0.018 \\
\hline Best radiological response, $\mathrm{n}(\%)$ & & & & $0(0.0)$ \\
\hline CR & $1(1.6)$ & $1(2.0)$ & $7(46.7)$ & \\
\hline PR & $10(15.6)$ & $8(16.3)$ & $7(46.7)$ & \\
\hline SD & $21(32.8)$ & $15(30.6)$ & $1(6.7)$ & \\
\hline PD & $32(50.0)$ & $25(51.0)$ & 46.7 & 0.041 \\
\hline Response rate (\%) & 17.2 & 18.4 & 93.3 & 0.003 \\
\hline Disease control rate (\%) & 50.0 & 49.0 & & \\
\hline
\end{tabular}

Data were presented as the number (percentage). *Statistical analysis was done between the latter half of sorafenib group and lenvatinib group. CR : complete response, HCC : hepatocellular carcinoma, PD : progressive disease, PR : partial response, SD : stable disease.

The ORR of lenvatinib was shown to be $38.5 \%-40.6 \%$ in previous studies $(7,26,27)$. A relatively high ORR of $61.3 \%$ was observed in a subgroup analysis of Japanese patients with intermediate-stage disease in the REFLECT study (28). These results were consistent with those of the present study. The reason for this increased ORR in the intermediate stage remains unclear. Further studies will be needed in order to determine the cause of this high ORR.

There were no significant differences in the overall survival between the lenvatinib group and the sorafenib group in the present study, as lenvatinib showed noninferiority compared to sorafenib in a phase III trial (7). Caution should be practiced in the interpretation of this overall survival analysis, as the number of patients was small and the median follow-up insufficient. Due to the small number of events, we were unable to conduct a multivariate analysis after adjusting for confounding factors. In addition, one of the problems in the present study was whether or not the ORR as assessed by mRECIST contributed to the overall survival. According to the landmark analysis of the REFLECT trial, the ORR was shown to be an independent predictor of the overall survival in advanced HCC patients, regardless of the treatment (29). Accordingly, a further study concerning whether or not first-line treatment with lenvatinib is superior to that with sorafenib for the overall survival should be conducted.

Treatment strategies for HCC change with the times. The concept of TACE failure/refractoriness was first reported in 2014 (2). Thus, we compared the percentage of TACE failure/refractoriness in intermediate-stage HCC patients between before and after publishing the concept. However, there were no significant differences in the percentage of TACE failure/refractoriness in sorafenib era. The emergence of lenvatinib encouraged physicians to convert from TACE to systemic chemotherapy earlier.

In conclusion, our findings showed that patients who satisfied the criteria of TACE failure/refractoriness were found more frequently in the lenvatinib group than in the sorafenib group in our analysis of intermediate HCC patients. The emergence of lenvatinib has encouraged physicians to convert TACE to systemic chemotherapy earlier in intermediate-stage HCC patients.

\section{Conflict of interest}

The authors declare no conflicts of interest in association with this study.

\section{References}

1. European Association for the Study of the Liver. EASL Clinical Practice Guidelines : Management of hepatocellular carcinoma. J. Hepatol., 2018, 69 : 182-236. [PMID : 29628281]

2. KUDO M, MATSUI O, IZUMI N, KADOYA M, OKUSAKA T. et al. Transarterial chemoembolization failure/refractoriness : JSH-LCSGJ criteria 2014 update. Oncology, 2014, 87 Suppl 1 : 22-31. [PMID : 25427730]

3. OGASAWARA S, CHIBA T, OOKA Y, KANOGAWA N, MOTOYAMA T. et al. Efficacy of sorafenib in intermediate-stage hepatocellular carcinoma patients refractory to transarterial chemoembolization. Oncology, 2014, 87 : 330-41. [PMID : 25227534]

4. ARIZUMI T, UESHIMA K, MINAMI T, KONO M, CHISHINA H., et al. Effectiveness of sorafenib in patients with transcatheter arterial chemoembolization (TACE) refractory and intermediate-stage hepatocellular carcinoma. Liver Cancer, 2015, 4 : 253-62. [PMID : 26734579]

5. LLOVET JM, RICCI S, MAZZAFERRO V, HILGARD P, GANE E. et al. Sorafenib in advanced hepatocellular carcinoma. N. Engl. J. Med., 2008, 359 : 378-90. [PMID : 18650514]

6. CHENG AL, KANG YK, CHEN Z, TSAO CJ, QIN S. et al. Efficacy and safety of sorafenib in patients in the Asia-Pacific region with advanced hepatocellular carcinoma : a phase III randomised, double-blind, placebocontrolled trial. Lancet Oncol., 2009, 10 : 25-34. [PMID : 19095497]

7. KUDO M, FINN RS, QIN S, HAN KH, IKEDA K. et al. Lenvatinib versus sorafenib in first-line treatment of patients with unresectable hepatocellular carcinoma : a randomised phase 3 non-inferiority trial. Lancet, 2018, 391 : 1163-73. [PMID : 29433850]

8. MATSUI J, FUNAHASHI Y, UENAKA T, WATANABE T, TSURUOKA A. et al. Multi-kinase inhibitor E7080 suppresses lymph node and lung metastases of human mammary breast tumor MDA-MB-231 via inhibition of vascular endothelial growth factor-receptor (VEGF-R) 2 and VEGF-R3 kinase. Clin Cancer Res 2008, 14 : 5459-65. [PMID : 18765537]

9. TOHYAMA O, MATSUI J, KODAMA K, HATA-SUGI N, KIMURA T et al. Antitumor activity of lenvatinib (e7080) : an angiogenesis inhibitor that targets multiple receptor tyrosine kinases in preclinical human thyroid cancer models. J. Thyroid. Res., 2014, 2014 : 638747. [PMID : 25295214]

10. YAMAMOTO Y, MATSUI J, MATSUSHIMA T, OBAISHI H, MIYAZAKI K. et al. Lenvatinib, an angiogenesis inhibitor targeting VEGFR/FGFR, shows broad antitumor activity in human tumor xenograft models associated with microvessel density and pericyte coverage. Vasc. Cell, 2014, 6 : 18. [PMID : 25197551]

11. BRUIX J, SHERMAN M. Management of hepatocellular carcinoma. Hepatology, 2005, 42 : 1208-36. [PMID : 16250051]

12. BOLONDI L, BURROUGHS A, DUFOUR JF, GALLE PR, MAZZAFERRO V. et al. Heterogeneity of patients with intermediate (BCLC B) Hepatocellular 
Carcinoma : proposal for a subclassification to facilitate treatment decisions. Semin. Liver Dis., 2012, 32 : 348-59. [PMID : 23397536]

13. JOHNSON PJ, BERHANE S, KAGEBAYASHI C, SATOMURA S, TENG M. et al. Assessment of liver function in patients with hepatocellular carcinoma : a new evidence-based approach-the ALBI grade. J. Cli. Oncol., 2015, 33 : 550-8. [PMID : 25512453]

14. LENCIONI R, LLOVET JM, HAN G, TAK WY, YANG J. et al. Sorafenib or placebo plus TACE with doxorubicin-eluting beads for intermediate stage HCC : The SPACE trial. J. Hepatol., 2016, 64 : 1090-8. [PMID : 26809111]

15. MEYER T, FOX R, MA YT, ROSS PJ, JAMES MW et al. Sorafenib in combination with transarterial chemoembolisation in patients with unresectable hepatocellular carcinoma (TACE 2) : a randomised placebocontrolled, double-blind, phase 3 trial. Lancet Gastroenterol. Hepatol., 2017, $2:$ 565-75. [PMID : 28648803]

16. YASUI Y, TSUCHIYA K, KUROSAKI M, TAKEGUCHI T, TAKEGUCHI Y. et al. Up-to-seven criteria as a useful predictor for tumor downstaging to within Milan criteria and Child-Pugh grade deterioration after initial conventional transarterial chemoembolization. Hepatol. Res., 2018, 48 : $442-$ 50. [PMID : 29278654]

17. HIRAOKA A, KUMADA T, KUDO M, HIROOKA M, KOIZUMI Y. et al. Hepatic function during repeated TACE procedures and prognosis after introducing sorafenib in patients with unresectable hepatocellular carcinoma : multicenter analysis. Dig. Dis., 2017, 35 : 602-10. [PMID : 29040999]

18. TAKADA J, HIDAKA H, NAKAZAWA T, KONDO M, NUMATA K. et al. Modified response evaluation criteria in solid tumors is superior to response evaluation criteria in solid tumors for assessment of responses to sorafenib in patients with advanced hepatocellular carcinoma. BMC Res. Notes, 2015, 8 : 609. [PMID : 26502722]

19. CHENG AL, THONGPRASERT S, LIM HY, SUKEEPAISARNJAROEN W, YANG TS. et al. Randomized, open-label phase 2 study comparing frontline dovitinib versus sorafenib in patients with advanced hepatocellular carcinoma. Hepatology, 2016, 64 : 774-84. [PMID : 27082062]

20. ARIZUMI T, UESHIMA K, CHISHINA H, KONO M, TAKITA M. et al. Duration of stable disease is associated with overall survival in patients with advanced hepatocellular carcinoma treated with sorafenib. Dig. Dis., 2014, 32 : 705-10. [PMID : 25376287]
21. HATANAKA T, KAKIZAKI S, UEHARA D, NAGASHIMA T, UENO T. et al. Impact of the prognostic nutritional index on the survival of Japanese patients with hepatocellular carcinoma treated with sorafenib : A multicenter retrospective study. Intern. Med., 2019, 58 : 1835-1844. [PMID : 30918170]

22. IRIE T, KURAMOCHI M, TAKAHASHI N. Dense accumulation of lipiodol emulsion in hepatocellular carcinoma nodule during selective balloonoccluded transarterial chemoembolization: measurement of balloonoccluded arterial stump pressure. Cardiovasc. Intervent. Radiol., 2013, 36 : 706-13. [PMID : 22996589]

23. HATANAKA T, ARAI H, SHIBASAKI M, TOJIMA H, TAKIZAWA D. et al. Factors predicting overall response and overall survival in hepatocellular carcinoma patients undergoing balloon-occluded transcatheter arteria chemoembolization : A retrospective cohort study. Hepatol. Res., 2018, 48 : 165-75. [PMID : 28500686]

24. LAMMER J, MALAGARI K, VOGL T, PILLEUL F, DENYS A. et al. Prospective randomized study of doxorubicin-eluting-bead embolization in the treatment of hepatocellular carcinoma : results of the PRECISION V study. Cardiovasc. Intervent. Radiol., 2010, 33 : 41-52. [PMID : 19908093]

25. GOLFIERI R, GIAMPALMA E, RENZULLI M, CIONI R, BARGELLINI I. et al. Randomised controlled trial of doxorubicin-eluting beads vs conventional chemoembolisation for hepatocellular carcinoma. Br. J. Cancer, 2014, $111:$ : 255-64. [PMID : 24937669]

26. OBI S, SATO T, SATO S, KANDA M, TOKUDOME Y. et al. The efficacy and safety of lenvatinib for advanced hepatocellular carcinoma in a realworld setting. Hepatol. Int., 2019, 13 : 199-204. [PMID : 30671808]

27. HIRAOKA A, KUMADA T, KARIYAMA K, TAKAGUCHI K, ATSUKAWA M. et al. Clinical features of lenvatinib for unresectable hepatocellular carcinoma in real-world conditions : Multicenter analysis. Cancer Med., 2019, $8:$ 137-46. [PMID : 30575325]

28. KUDO M. Extremely high objective response rate of lenvatinib : its clinical relevance and changing the treatment paradigm in hepatocellular carcinoma Liver Cancer, 2018, 7 : 215-24. [PMID : 30319981]

29. KUDO M, FINN RS, QIN S, HAN K-H, IKEDA K. et al. Analysis of survival and objective response (OR) in patients with hepatocellular carcinoma in phase III study of lenvatinib (REFLECT). Journal of Clinical Oncology, $2019,37: 186$. 